\title{
Super-Resolution Raman Spectroscopy by Digital Image Processing
}

\section{Motohiro Tomita, ${ }^{1,2}$ Hiroki Hashiguchi, ${ }^{1}$ Takuya Yamaguchi, ${ }^{1}$ Munehisa Takei, ${ }^{1,2}$ Daisuke Kosemura, ${ }^{1}$ and Atsushi Ogura ${ }^{1}$}

\author{
${ }^{1}$ School of Science and Technology, Meiji University, 1-1-1 Higashimita, Tama-ku, Kawasaki 214-8571, Japan \\ ${ }^{2}$ Research Fellow of the Japan Society for the Promotion of Science, 8 Ichibancho, Chiyoda, \\ Tokyo 102-8472, Japan
}

Correspondence should be addressed to Motohiro Tomita; mot_tom@meiji.ac.jp

Received 5 October 2012; Revised 6 January 2013; Accepted 1 February 2013

Academic Editor: Kong-Thon Tsen

Copyright (C) 2013 Motohiro Tomita et al. This is an open access article distributed under the Creative Commons Attribution License, which permits unrestricted use, distribution, and reproduction in any medium, provided the original work is properly cited.

\begin{abstract}
We demonstrate the results of a strain (stress) evaluation obtained from Raman spectroscopy measurements with the superresolution method (the so-called super-resolution Raman spectroscopy) for a Si substrate with a patterned SiN film (serving as a strained Si sample). To improve the spatial resolution of Raman spectroscopy, we used the super-resolution method and a highnumerical-aperture immersion lens. Additionally, we estimated the spatial resolution by an edge force model (EFM) calculation. One- and two-dimensional stress distributions in the Si substrate with the patterned SiN film were obtained by super-resolution Raman spectroscopy. The results from both super-resolution Raman spectroscopy and the EFM calculation were compared and were found to correlate well. The best spatial resolution, $70 \mathrm{~nm}$, was achieved by super-resolution Raman measurements with an oil immersion lens. We conclude that super-resolution Raman spectroscopy is a useful method for evaluating stress in miniaturized state-of-the-art transistors, and we believe that the super-resolution method will soon be a requisite technique.
\end{abstract}

\section{Introduction}

Raman spectroscopy is used as a stress evaluation method for strained $\mathrm{Si}$, which is a technique for improving device performance. State-of-the-art metal-oxide-semiconductor fieldeffect transistors (MOSFETs) with strained Si have been scaled down and have become complicated. There is a significant demand to measure the strain induced in such $\mathrm{Si}$ nanodevices, because the electrical properties of transistors considerably depend on the strain. Various methods of nanoscale strain (stress) evaluation have been demonstrated, including convergent-beam electron diffraction (CBED), micro-Raman spectroscopy, and micro-X-ray diffraction (XRD) [1-5]. The Raman spectroscopy is advantageous because it permits the nondestructive and precise measurement of stress in $\mathrm{Si}$ with relatively high spatial resolution. In the previous study, the spatial resolution of Raman spectroscopy was improved by the use of a high-numerical- aperture (NA) immersion lens $[6,7]$. However, the spatial resolution of current Raman measurements is insufficient to evaluate state-of-the-art MOSFETs, because the spatial resolution of an optical measurement cannot far exceed its wavelength due to the diffraction limit. The data diffusion is attributed to a variation in the probe shape, which is determined by mirrors, filters, and lenses on light path; the probe diameter especially was varied by NA of an objective lens. Generally, that is inevitable in optical evaluation techniques. Accordingly, digital processing technology is necessary to solve this problem. The digital processing technology of the super-resolution method permits the evaluation of the spatial resolution beyond the optical diffraction limit.

In this study, we improved the spatial resolution of Raman spectroscopy by the use of the super-resolution method and a high NA immersion lens. The super-resolution method is the digital technique of improving for spatial resolution. 


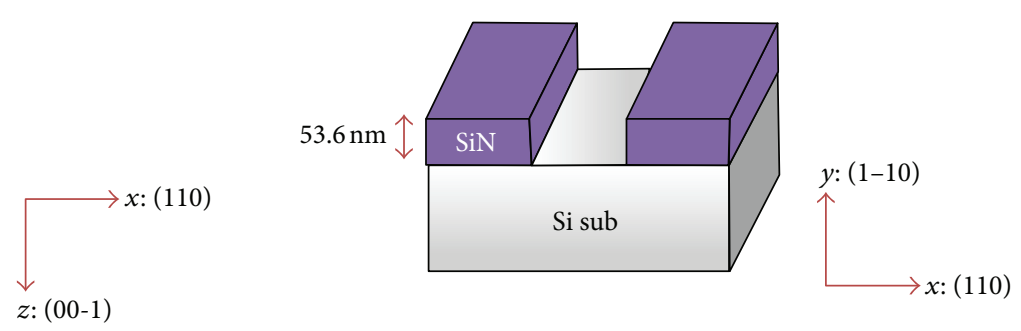

(a) bird's-eye view

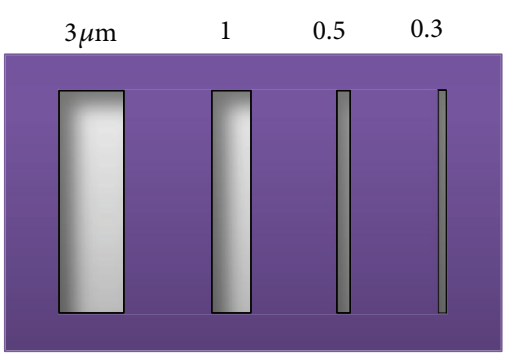

(b) plan view

FIGURE 1: (a) Cross-sectional view of Si with patterned SiN structures and (b) plan views of etched L\&S pattern.

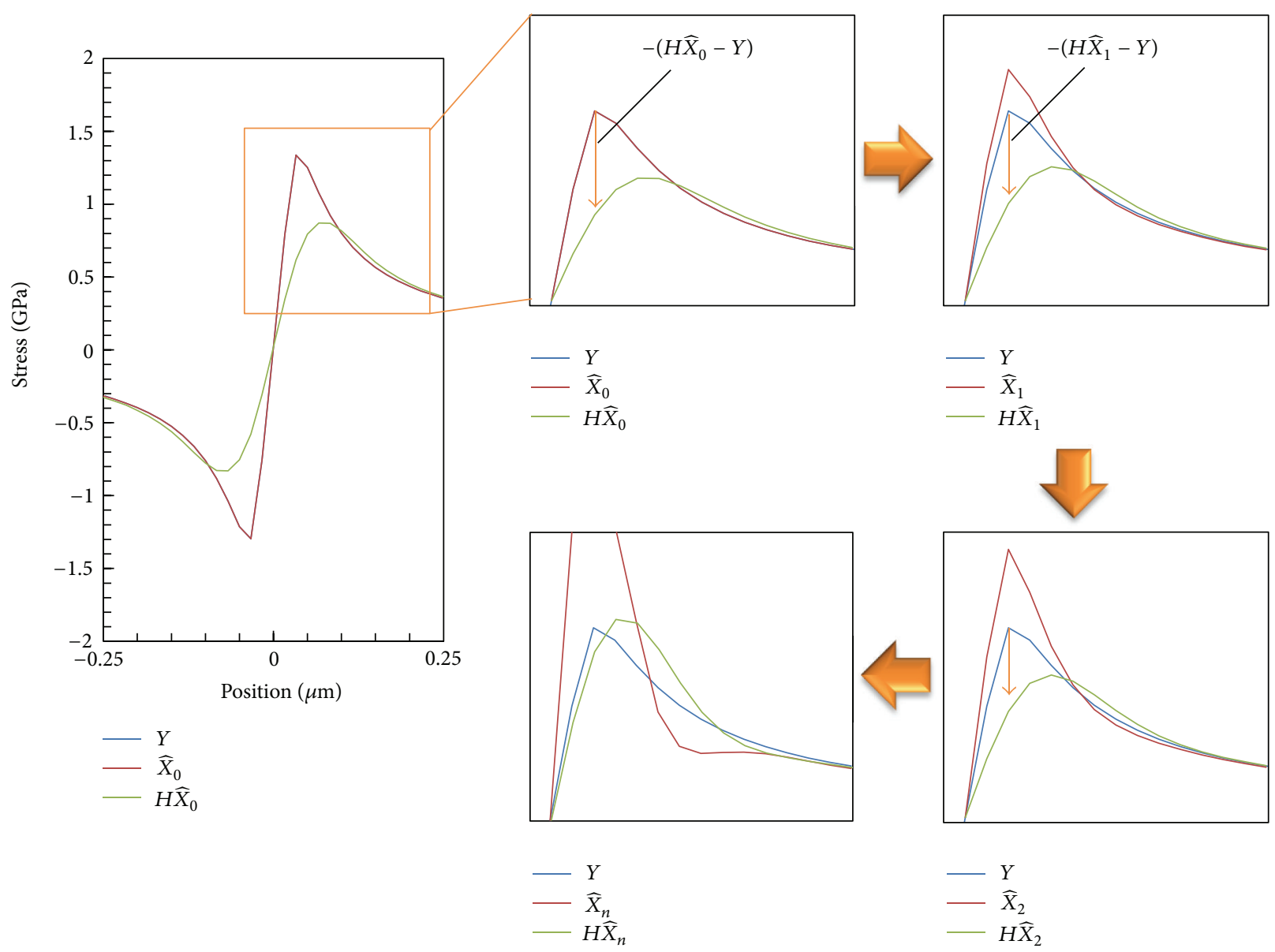

FIGURE 2: The every steps of the recurrence formula of BTV deconvolution method.

It is possible to combine the super-resolution method with other techniques of improving spatial resolution. Moreover, we demonstrated results obtained by Raman spectroscopy measurements with the super-resolution method for a $\mathrm{Si}$ substrate with a patterned SiN film. The SiN films are used in the state-of-the-art MOSFETs to enhance the electron and hole mobilities. Additionally, we checked the validation of the super-resolution algorithm and estimated the spatial resolution by edge force model (EFM) calculation.

\section{Experimental Procedure}

2.1. Samples. To evaluate the spatial resolution of Raman spectroscopy, a Si substrate with a patterned SiN film was used as a strained Si sample. The structures of the samples are shown in Figure 1. The thickness of the SiN film was $53.6 \mathrm{~nm}$. The inner stress of the $\mathrm{SiN}$ film was approximately $-2.5 \mathrm{GPa}$ of the compressive stress estimated in this study. Line/space (L\&S) patterns were formed by electron beam lithography 


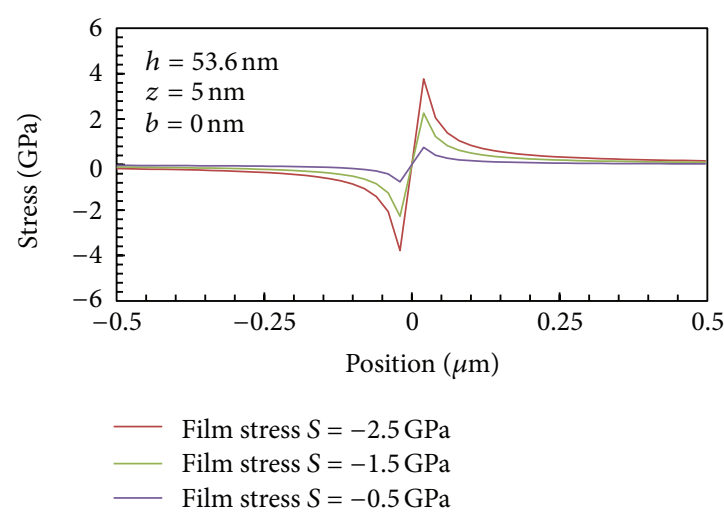

(a)

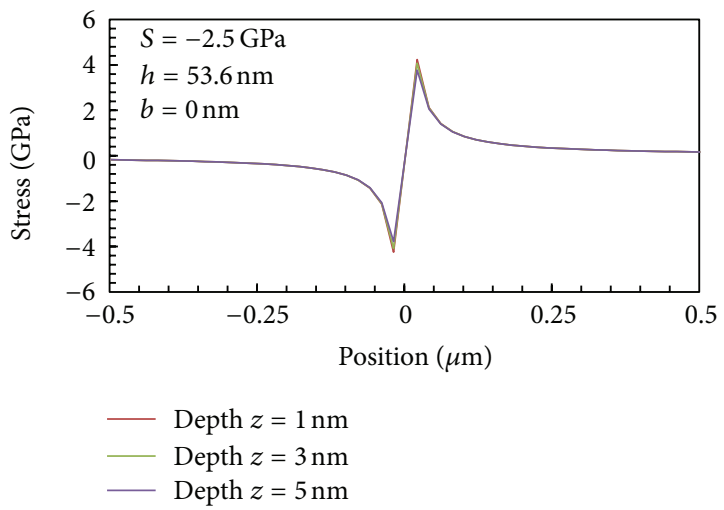

(c)

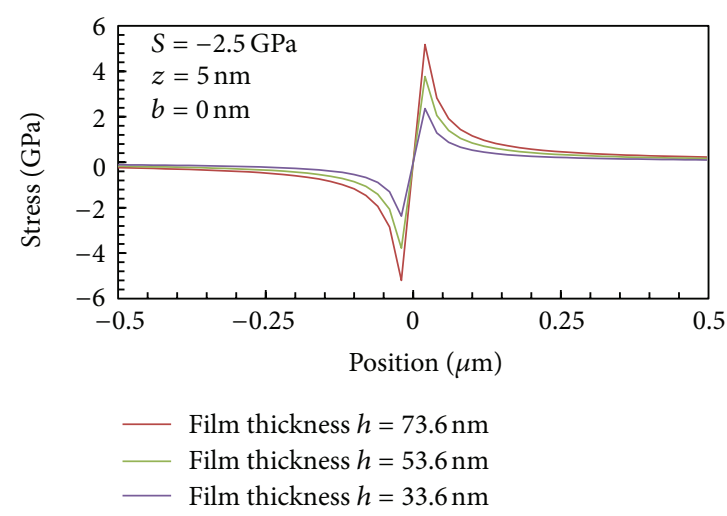

(b)

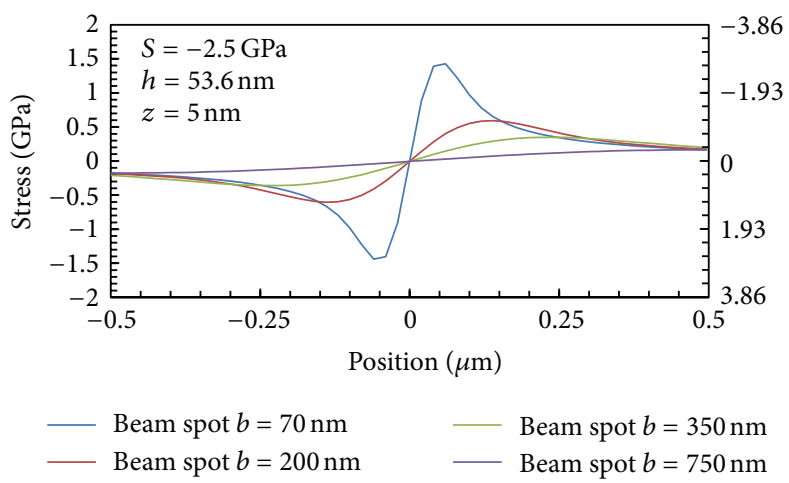

(d)

Figure 3: The stress dependences on (a) $S_{\text {Film }}$ of the film stress, (b) $h_{\text {Film }}$ of the film thickness, (c) $z$ of the detection depth, and (d) $b$ of the beam spot size.

and reactive ion etching. The space widths in the L\&S pattern were $3,1,0.5$, and $0.3 \mu \mathrm{m}$, as shown in Figure 1(a). The line length and the interval between lines were 150 and $20 \mu \mathrm{m}$, respectively. The coordinate system is also shown in Figure 1 $(x:(110), y:(1-10)$, and $z:(00-1))$.

2.2. Methods of Stress/Strain Evaluation. UV-Raman spectroscopy was performed to obtain stress distributions. An Arion laser $(\lambda=364 \mathrm{~nm})$ was used as an excitation source, and its penetration depth into $\mathrm{Si}$ was approximately $5 \mathrm{~nm}$. The $\mathrm{SiN}$ film is transparent to the wavelength of $364 \mathrm{~nm}[8,9]$. A conventional lens $(\mathrm{NA}=0.5)$ and a high NA immersion lens $(\mathrm{NA}=1.4)$ were used. The scan step sizes were 200 and $100 \mathrm{~nm}$ in the conventional lens and the immersion lens condition. A detailed explanation of the stress measurement by UV-Raman spectroscopy is given in $[8,9]$. The superresolution method was applied to Raman spectroscopy (the so-called super-resolution Raman spectroscopy) to obtain extremely high spatial resolution. In super-resolution Raman spectroscopy, the bilateral total variation (BTV) deconvolution method was selected so that noise enhancement could be suppressed during the calculation [10-14]. In a previous study, it was reported that there was a good correlation between the stress calculated by the EFM and that measured by the Raman method with corrections for detection depth and beam spot size (in this case, the beam spot profile corresponded to the diffusion filter $H$ ) [15-17]. In this report, a Gaussian filter was used as diffusion filter $H$. Thus, the restored data is obtained using a Gaussian filter as $H$. The BTV method is defined by

$$
\begin{aligned}
\widehat{X}=\arg \min _{x}[ & \lambda_{0}\|H X-Y\|+\lambda_{1} J_{1}(X) \\
& \left.+\lambda_{2} J_{2}(X)+\lambda_{3} J_{3}(X)\right],
\end{aligned}
$$

where $Y$ indicates the observed data, $\widehat{X}$ indicates the predicted original data, which was not affected by the diffusion, and $J_{1}, J_{2}, J_{3}$, and $\lambda$ indicate a bilateral filter, a median filter, a Laplacian filter, and the weighting factor of each filter, respectively. In the actual procedure, (1) is achieved by

$$
\begin{aligned}
& \widehat{X}_{0}=Y, \\
& \widehat{X}_{n+1}=\widehat{X}_{n}-\left\lfloor\lambda_{0}\left\|H \widehat{X}_{n}-Y\right\|+\lambda_{1} J_{1}\left(\widehat{X}_{n}\right)\right. \\
& \\
& \left.\quad+\lambda_{2} J_{2}\left(\widehat{X}_{n}\right)+\lambda_{3} J_{3}\left(\widehat{X}_{n}\right)\right\rfloor .
\end{aligned}
$$

Figure 2 shows the every steps of (2). The recurrence formula of (2) was repeatedly executed. Then, it was stopped when the difference between $\widehat{X}_{n}$ and $\widehat{X}_{n+1}$ became sufficiently small. In deconvolution, we adjusted the beam spot parameter $\sigma_{0}$ of $H$ to the experimental condition. We use the parameters $\sigma_{0}$ $=1100$ and 400 for the conventional lens and the immersion lens condition. A detailed explanation of the BTV method is given in [11]. 


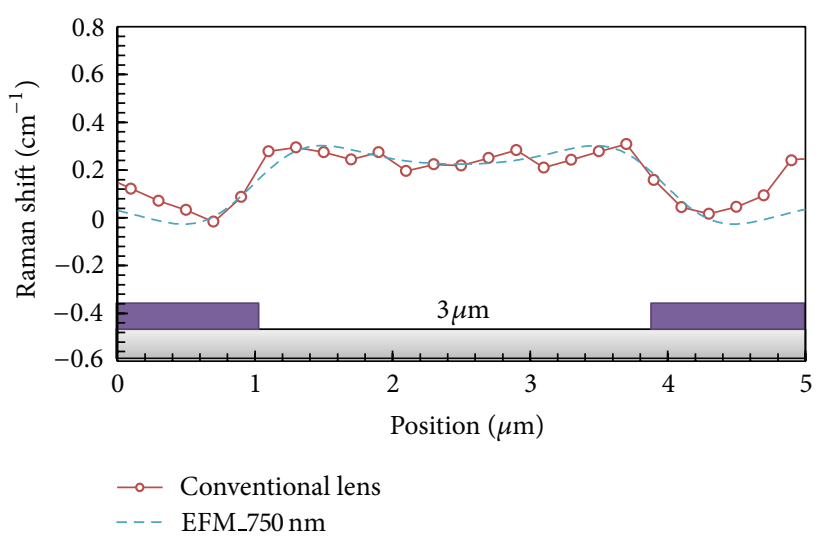

(a)

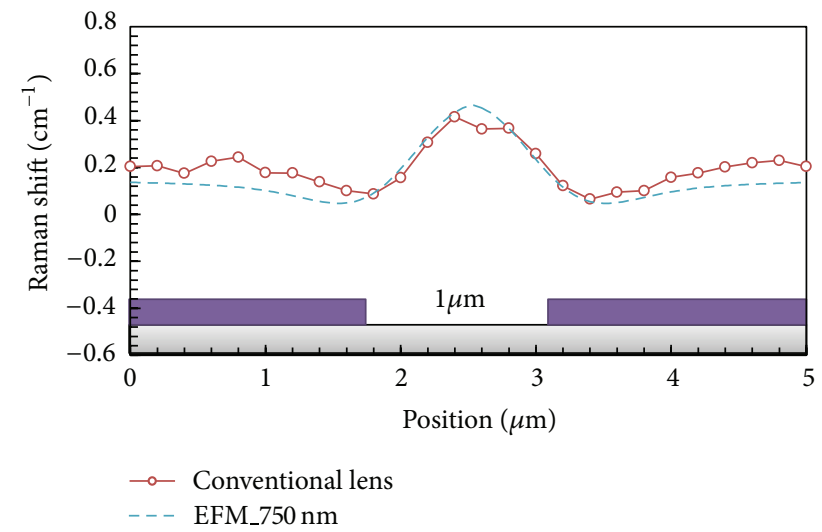

(b)

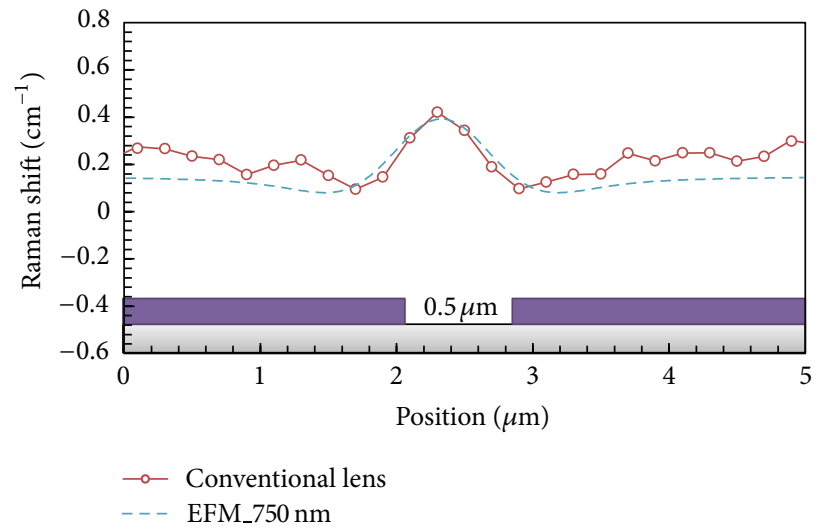

(c)

FIGURE 4: One-dimensional profiles of Raman shifts in the center of L\&S pattern obtained by the Raman spectroscopy and EFM calculation with conventional lens (in space widths of (a) 5, (b) 3, and (c) $1 \mu \mathrm{m}$ ).

The EFM with corrections for detection depth and beam spot size $[10,11]$ was employed to check the validation and to estimate the spatial resolution before and after super resolution. In this model, it is assumed that the stresses are concentrated to film edge and the edge forces are dependent on film thickness and stress $[18,19]$. The calculated stress profiles in the $\mathrm{Si}$ substrate with the L\&S pattern were estimated by this model. It is necessary to convert the stress to the Raman shift. The uniaxial stress assumption was used because the Raman shift of the vertical and shear stresses was sufficiently smaller than that of the uniaxial stress in this sample. Thus, the vertical and shear stresses were ignored in this study. The uniaxial stress and Raman shift conversion factor is -1.93 . Figure 3 shows the stress dependences on $S_{\text {Film }}$ of the film stress, $h_{\text {Film }}$ of the film thickness, $z$ of the detection depth, and $b$ of the beam spot size. We performed curve fitting of the least-square approach between the measurement data and the EFM calculation with the corrections, with the fitting parameter of $b$.

\section{Results and Discussion}

3.1. Spatial Resolution Improvement by an Oil Immersion Lens. Figures 4 and 5 show one-dimensional profiles of Raman wavenumber shifts for the Si substrate with a patterned SiN film (with space widths of 3, 1, and $0.5 \mu \mathrm{m}$ ). Solid and dashed lines show the results of Raman spectroscopy and the EFM calculation, respectively. Oil-immersion and conventional objective lenses were also compared in a previous study [7]. However, they were compared on shallow trench isolated Si. Moreover, we used the EFM calculations and estimated the spatial resolution by the least-square approach fitting. In the EFM calculation, we used the parameters $S_{\text {Film }}=-2.5 \mathrm{GPa}$, $h_{\mathrm{Film}}=53.6 \mathrm{~nm}$, and $z=5 \mathrm{~nm}$. The parameter $b$ was 750 and $350 \mathrm{~nm}$ when using the conventional and oil immersion lenses, respectively. Comparing the results of the Raman measurements with the conventional and oil immersion lenses in Figures 4 and 5, the stress enhancement at the $\mathrm{SiN}$ film edges is clearer in the oil immersion Raman method than in the conventional Raman method. Furthermore, it was observed that the strain induced in the space region increases with reducing space width, specifically in the oil immersion Raman measurement, which confirms the strain-size effect as was observed in previous studies [20,21]. As a result, the beam spot sizes were determined to be 750 and $350 \mathrm{~nm}$ in conventional and oil immersion Raman spectroscopy, respectively, obtained by comparing the measured data and the EFM calculations with the correction of optical penetration depth and beam spot size. The spatial resolution was improved using the oil immersion lens. Additionally, the $S_{\text {Film }}$ of the film 


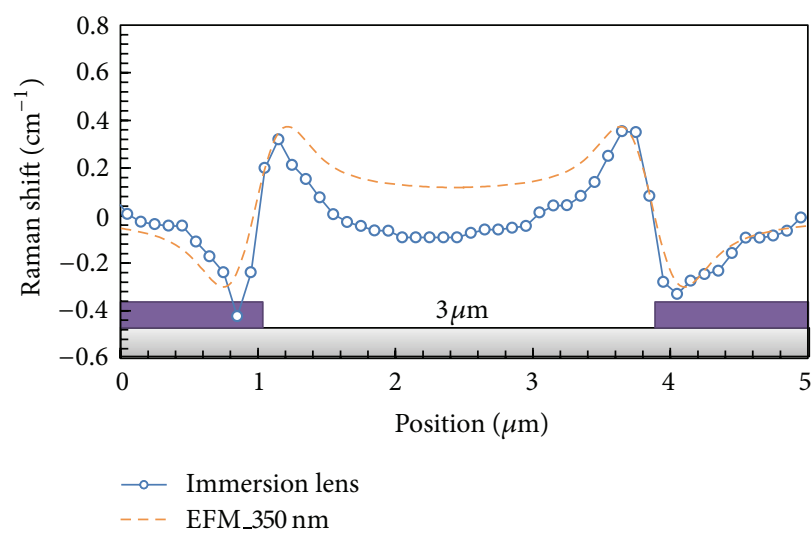

(a)

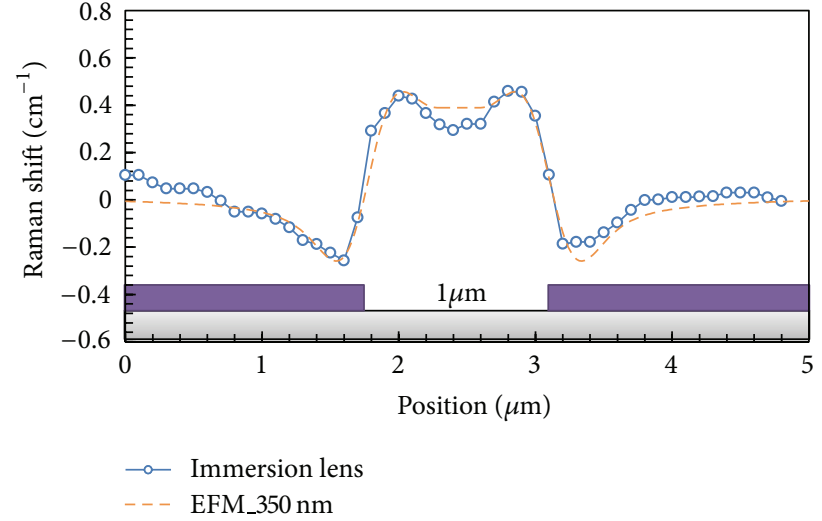

(b)

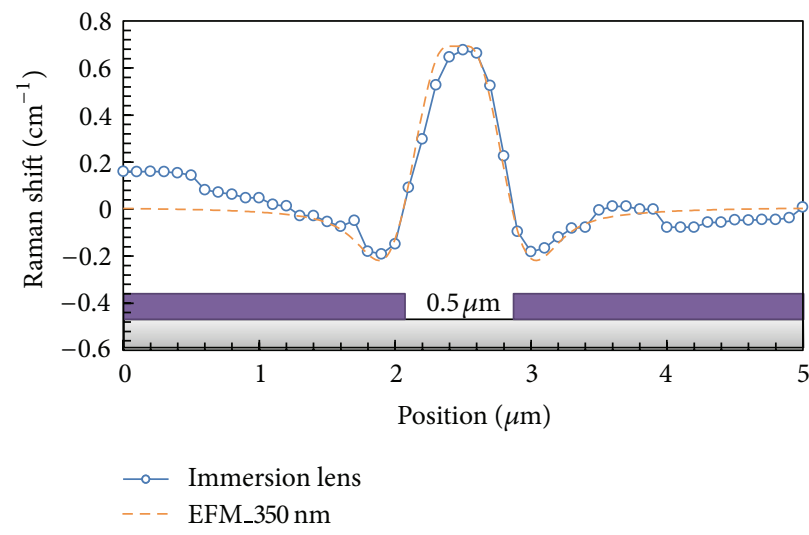

(c)

FIGURE 5: One-dimensional profiles of the Raman shifts in the center of L\&S pattern obtained by the Raman spectroscopy and EFM calculation with immersion lens (in space widths of (a) 5 , (b) 3 , and (c) $1 \mu \mathrm{m}$ ).

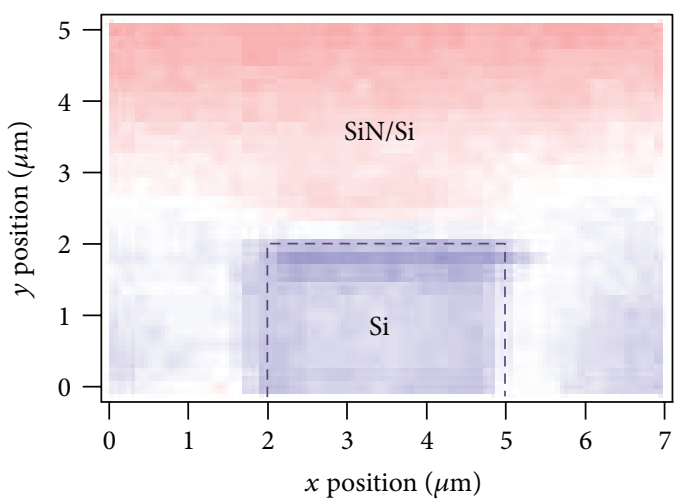

(a)

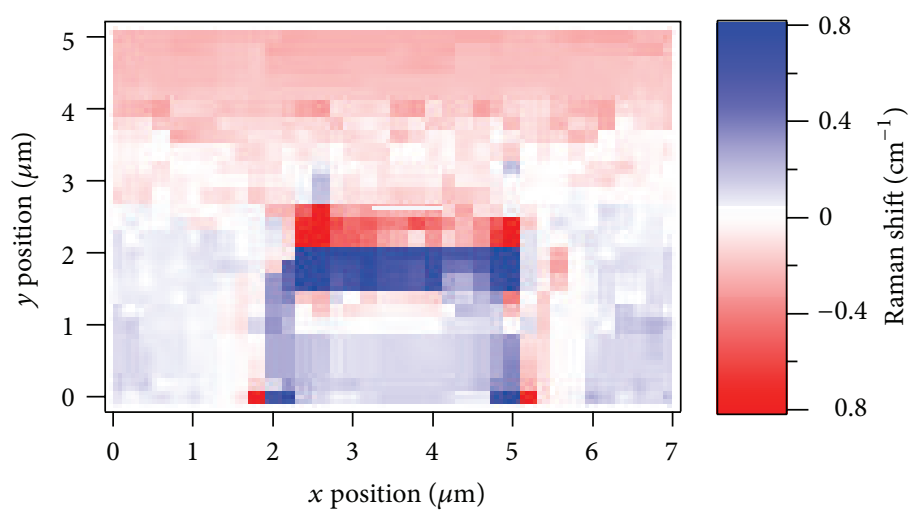

(b)

FiguRE 6: Two-dimensional distributions of the Raman shifts in the end of L\&S pattern obtained by (a) conventional and (b) super-resolution Raman measurements with conventional lens.

stress was estimated at $-2.5 \mathrm{GPa}$ by EFM fitting. Therefore, it is expected that the SiN film has high film density, because the film stress is highly compressive [22].

3.2. Super-Resolution Raman Spectroscopy. Figure 6 shows the two-dimensional distribution of Raman shifts at the end of the line/space SiN pattern sample ( $3 \mu \mathrm{m}$ space width) obtained by (a) conventional and (b) super-resolution Raman measurements with a conventional lens. In Figure 6, blue and red colors indicate the compressive and tensile strain, respectively. The super-resolution image (Figure 6(b)) was obtained by deconvolving the observed data (Figure 6(a)) 


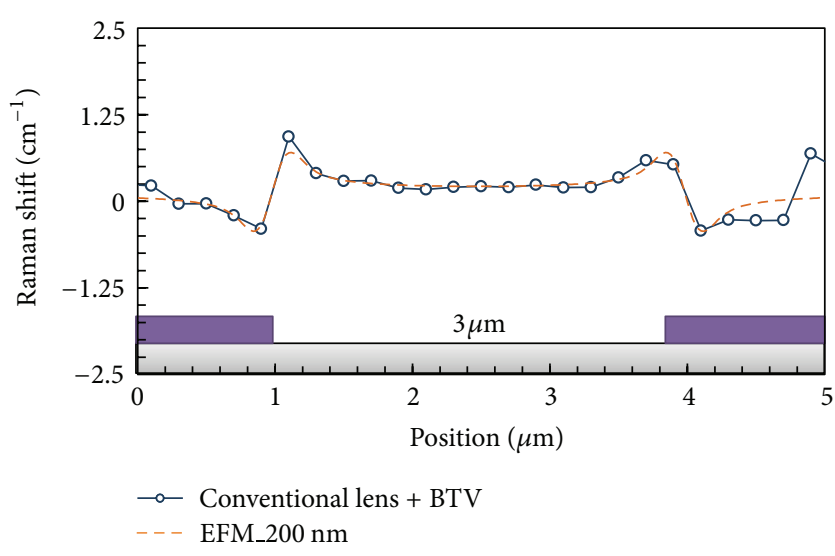

(a)

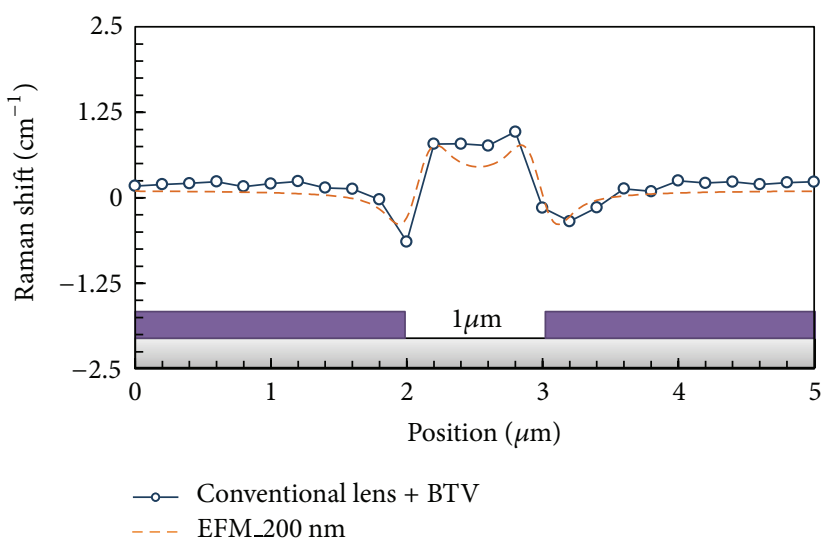

(b)

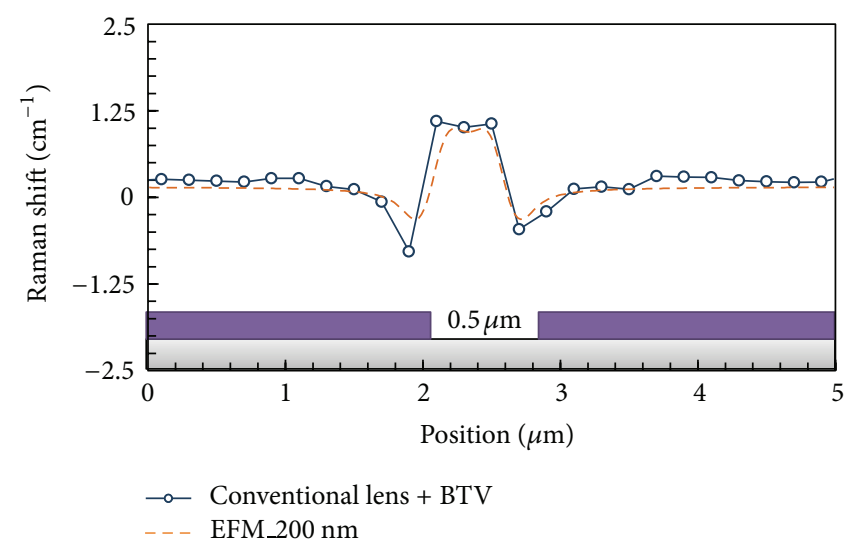

(c)

FIGURE 7: One-dimensional profiles of Raman shifts in the center of L\&S pattern obtained by super-resolution Raman spectroscopy and EFM calculation with conventional lens (in space widths of (a) 5 , (b) 3 , and (c) $1 \mu \mathrm{m}$ ).

using this $H$ filter, which was extended to two dimensions. In deconvolution procedure, the data stop was 3 points away from the inner edge of the measured area. The specific Raman shift map contrast appears in super-resolution Raman spectroscopy. The deconvolution method can be accurately applied to the two-dimensional evaluation. Figure 7 shows one-dimensional profiles of Raman shifts for the Si substrate with a patterned $\mathrm{SiN}$ film obtained by super-resolution Raman spectroscopy and the EFM calculation with a conventional lens. In the EFM calculation, we used the parameters $S_{\text {Film }}=-2.5 \mathrm{GPa}, h_{\text {Film }}=53.6 \mathrm{~nm}, z=5 \mathrm{~nm}$, and $b=$ $200 \mathrm{~nm}$. The stress enhancement at the SiN film edges and the strain-size effect were clearly observed after deconvolution, compared to the results without deconvolution (Figure 4). Moreover, stress relaxation at the center of the space region was confirmed even for the $0.5 \mu \mathrm{m}$ wide pattern. As a result, there is good correlation between the results of superresolution Raman spectroscopy and the EFM calculation. The spatial resolution of super-resolution Raman spectroscopy improved from $750 \mathrm{~nm}$ to $200 \mathrm{~nm}$. This value is as good as $350 \mathrm{~nm}$ obtained by oil immersion lens condition. As a result, the resolution improvement of deconvolution method with conventional lens is effectively the same as in the oil immersion lens condition.
Figure 8 shows one-dimensional profiles of Raman wavenumber shifts for the $\mathrm{Si}$ substrate with a patterned SiN film (with space widths of 3,1 , and $0.5 \mu \mathrm{m}$ ) by superresolution Raman spectroscopy with an oil immersion lens and the EFM calculation. In the EFM calculation, we used the parameters $S_{\text {Film }}=-2.5 \mathrm{GPa}, h_{\text {Film }}=53.6 \mathrm{~nm}, z=5 \mathrm{~nm}$, and $b=70 \mathrm{~nm}$. The stress enhancement at the $\mathrm{SiN}$ film edges was emphasized to a greater degree. Very high tensile and compressive stress of a maximum of $1.3 \mathrm{GPa}$ was obtained. Stress relaxation at the center of the space region was also clearly confirmed at the $0.5 \mu \mathrm{m}$ wide pattern, the same as in using a conventional lens and super resolution. As a result, the spatial resolution of the super-resolution Raman measurements with the oil-immersion lens was improved from $350 \mathrm{~nm}$ to $70 \mathrm{~nm}$ by the use of deconvolution and the oil immersion lens. We consider that a spatial resolution of $70 \mathrm{~nm}$ is sufficient for the stress evaluation of state-of-the-art scaled-down MOSFETs.

Figure 9 shows one-dimensional profiles of Raman wavenumber shifts for the $\mathrm{Si}$ substrate with a patterned SiN film $(0.3 \mu \mathrm{m}$ space width) by conventional and superresolution Raman spectroscopy with (a) conventional and (b) immersion lenses compared with the EFM calculation. In Figure 9(a), stress enhancement was not observed, because 


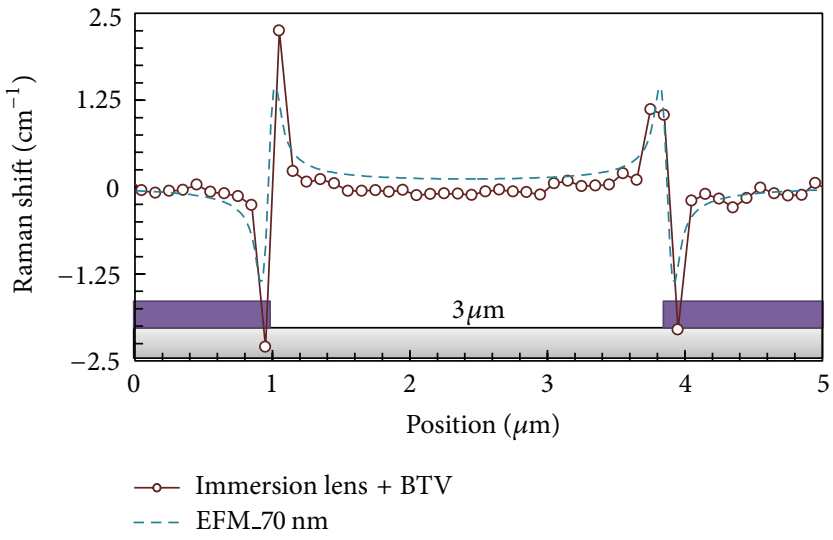

(a)

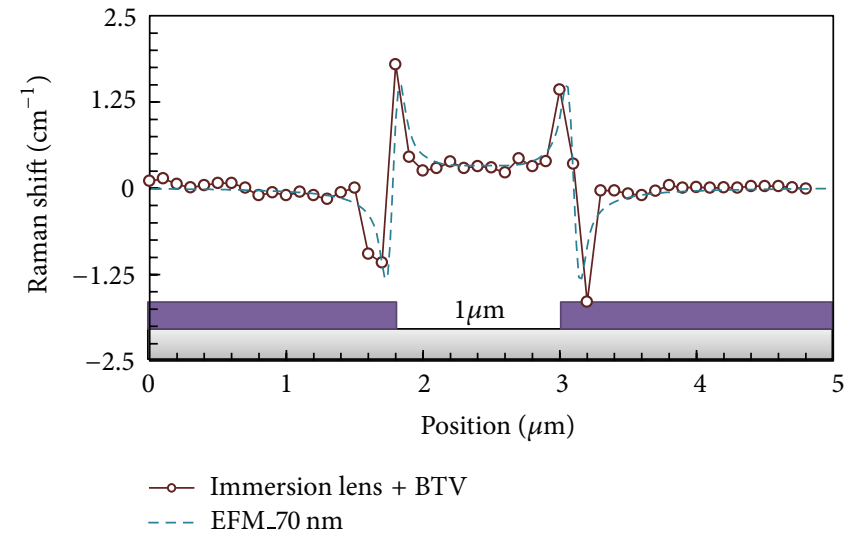

(b)

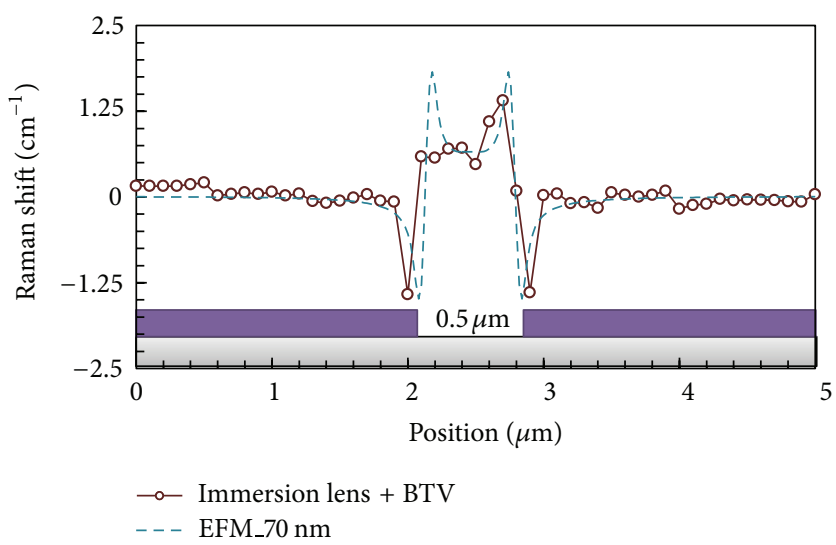

(c)

FIGURE 8: One-dimensional profiles of Raman shifts in the center of L\&S pattern obtained by super-resolution Raman spectroscopy and EFM calculation with immersion lens (in space widths of (a) 5 , (b) 3 , and (c) $1 \mu \mathrm{m}$ ).

the signal-to-noise ratio before the super-resolution treatment was low and close to the intensity of the noise. The Raman shift profiles were assumed to be noise caused by the super-resolution algorithm. On the other hand, stress enhancement was clearly observed in Figure 9(b). To obtain clear super-resolution Raman profiles, a high signal-to-noise ratio is required in the observed data. However, the superresolution technique is advantageous because it is not influenced by the sample because it includes digital processing. If we can solve the signal-to-noise ratio problem, superresolution Raman spectroscopy will be a powerful technique for nanoscale stress evaluation.

\section{Conclusions}

In this paper, the spatial resolution of Raman spectroscopy was improved by the use of digital processing technology provided by the super-resolution method, along with a highnumerical-aperture lens. A Si substrate with a patterned SiN film was used as a strained $\mathrm{Si}$ sample. The digital processing enabled us to evaluate a sharp Raman shift distribution, and very high tensile and compressive stress of a maximum of $1.3 \mathrm{GPa}$ was obtained. Moreover, to evaluate the spatial resolution of Raman spectroscopy, an EFM calculation with corrections for detection depth and beam spot size was used for curve fitting. As a result, correlation between the results of super-resolution Raman spectroscopy and the EFM calculation was good. The best spatial resolution, $70 \mathrm{~nm}$, was achieved by the super-resolution Raman measurements with the oil immersion lens. This value was an improvement over the $350 \mathrm{~nm}$ obtained by a conventional oil immersion Raman measurement and was approximately 0.093 times the $750 \mathrm{~nm}$ obtained by a conventional measurement. The spatial resolution of $70 \mathrm{~nm}$ is close to the size of the stateof-the-art MOSFETs. We consider super-resolution Raman spectroscopy to be very useful for nanoscale stress evaluation. However, the profile with the low signal-to-noise ratio was not improved by the super-resolution algorithm, because the Raman shift profiles were assumed to be noise caused by the super-resolution algorithm. It is necessary that we obtain the observed data with a high signal-to-noise ratio. The super-resolution technique is advantageous because it is not influenced by the sample since it includes digital processing. If we can solve the signal-to-noise ratio problem, super-resolution Raman spectroscopy will be a powerful technique for nanoscale stress evaluation. We believe that 


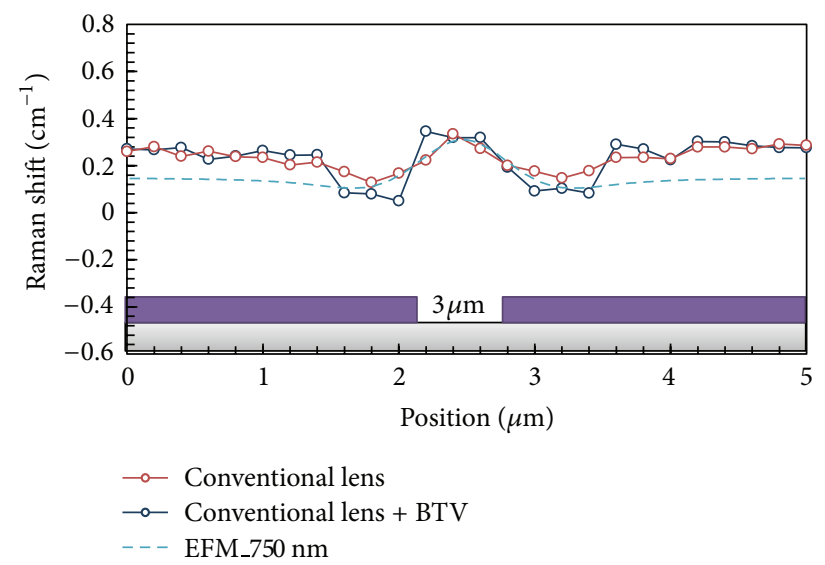

(a)

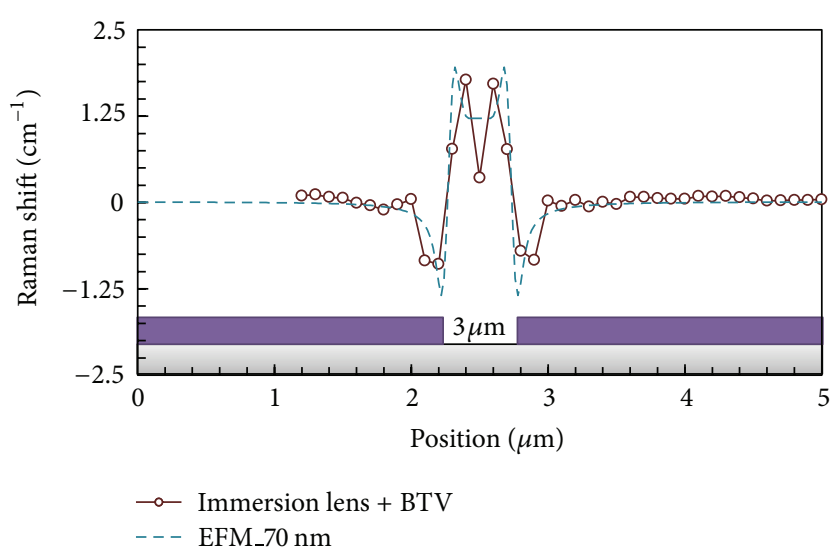

(b)

FIGURE 9: One-dimensional profiles of Raman wavenumber shifts for the Si substrate with a patterned SiN film $(0.3 \mu \mathrm{m}$ space width) by conventional and super-resolution Raman spectroscopy with (a) conventional and (b) immersion lenses compared with the EFM calculation.

the super-resolution method will be indispensable to for evaluating future MOSFETs, which will surely surpass the present ones in complexity.

\section{Acknowledgment}

This study was partly supported by the Semiconductor Technology Academic Research Center (STARC).

\section{References}

[1] V. Senez, A. Armigliato, I. De Wolf et al., "Strain determination in silicon microstructures by combined convergent beam electron diffraction, process simulation, and micro-Raman spectroscopy," Journal of Applied Physics, vol. 94, no. 9, pp. 55745583, 2003.

[2] H. C. Floresca, J. G. Wang, M. J. Kim, and J. A. Smythe, "Shallow trench isolation liners and their role in reducing lattice strains," Applied Physics Letters, vol. 93, no. 14, Article ID 143116, 2008.

[3] A. Parikh, W. Yarbrough, M. Mason, S. Sridhar, P. R. Chidambaram, and Z. Cai, "Characterization of structure and morphology of an advanced p-channel field effect transistor under uniaxial stress by synchrotron X-ray diffraction," Applied Physics Letters, vol. 90, Article ID 172117, 2007.

[4] H. Yan, C. E. Murray, and I. C. Noyan, "Mapping local strain in thin film/substrate systems using X-ray microdiffraction topography," Applied Physics Letters, vol. 90, no. 9, Article ID 091918, 2007.

[5] M. Gailhanou, A. Loubens, J. S. Micha et al., "Strain field in silicon on insulator lines using high resolution X-ray diffraction," Applied Physics Letters, vol. 90, no. 11, Article ID 111914, 2007.

[6] V. Poborchii, T. Tada, and T. Kanayama, "High-spatialresolution Raman microscopy of stress in shallow-trenchisolated Si structures," Applied Physics Letters, vol. 89, Article ID 233505, 2006

[7] I. De Wolf, G. Groeseneken, H. E. Meas et al., "MicroRaman spectroscopy evaluation of the local mechanical stress in shallow trench isolation CMOS structures: correlation with defect generation and diode leakage," in Proceedings ofthe 24th
International Symposium for Testing and Failure Analysis (ISTFA '98), p. 11, Dallas, Tex, USA, November 1998.

[8] A. Ogura, T. Yoshida, D. Kosemura et al., "Evaluation of supercritical thickness strained-Si on insulator (sc-SSOI) substrate," Solid-State Electronics, vol. 52, no. 12, pp. 1845-1848, 2008.

[9] A. Ogura, K. Yamasaki, D. Kosemura, S. Tanaka, I. Chiba, and R. Shimidzu, "UV-Raman spectroscopy system for local and global strain measurements in Si," Japanese Journal of Applied Physics, vol. 45, no. 4B, pp. 3007-3011, 2006.

[10] M. Tomita, D. Kosemura, M. Takei, K. Nagata, H. Akamatsu, and A. Ogura, "Evaluation of strained silicon by electron back scattering pattern compared with Raman measurement and edge force model calculation," Key Engineering Materials, vol. 470, pp. 123-128, 2011.

[11] M. Tomita, D. Kosemura, M. Takei, K. Nagata, H. Akamatsu, and A. Ogura, "Evaluation of strained-silicon by electron backscattering pattern measurement: comparison study with UV-raman measurement and edge force model calculation," Japanese Journal of Applied Physics, vol. 50, Article ID 010111, 2011.

[12] S. Farsiu, M. D. Robinson, M. Elad, and P. Milanfar, "Fast and robust multiframe super resolution," IEEE Transactions on Image Processing, vol. 13, no. 10, pp. 1327-1344, 2004.

[13] H. Takeda, S. Farsiu, and P. Milanfar, "Deblurring using regularized locally adaptive Kernel regression," IEEE Transactions on Image Processing, vol. 17, no. 4, pp. 550-563, 2008.

[14] T. F. Chan and C. K. Wong, "Total variation blind deconvolution," IEEE Transactions on Image Processing, vol. 7, no. 3, pp. 370-375, 1998.

[15] I. De Wolf, H. E. Maes, and S. K. Jones, "Stress measurements in silicon devices through Raman spectroscopy: bridging the gap between theory and experiment," Journal of Applied Physics, vol. 79, no. 9, p. 7148, 1996.

[16] I. De Wolf, "Micro-Raman spectroscopy to study local mechanical stress in silicon integrated circuits," Semiconductor Science and Technology, vol. 11, no. 2, p. 139, 1996.

[17] I. De Wolf, J. Vanhellemont, A. Romano-Rodriguez, H. Norstrom, and H. E. Maes, "Micro-Raman study of stress distribution in local isolation structures and correlation with transmission electron microscopy," Journal of Applied Physics, vol. 71, no. 2, p. 898, 1992. 
[18] S. M. Hu, "Film-edge-induced stress in substrates," Journal of Applied Physics, vol. 50, no. 7, p. 4661, 1979.

[19] S. M. Hu, "Stress-related problems in silicon technology," Journal of Applied Physics, vol. 70, no. 6, p. R53, 1991.

[20] D. Kosemura, Y. Kakemura, T. Yoshida et al., "Characterization of strain for high-performance metal-oxide-semiconductor field-effect-transistor," Japanese Journal of Applied Physics, vol. 47, no. 4, pp. 2538-2543, 2008.

[21] M. Takei, D. Kosemura, K. Nagata et al., "Channel strain analysis in high-performance damascene-gate p-metal-oxidesemiconductor field effect transistors using high-spatial resolution Raman spectroscopy," Journal of Applied Physics, vol. 107, no. 12, Article ID 124507, 2010.

[22] A. Ogura, H. Saitoh, D. Kosemura et al., "Evaluation and control of strain in si induced by patterned SiN stressor," Electrochemical and Solid-State Letters, vol. 12, no. 4, pp. H117H119, 2009. 

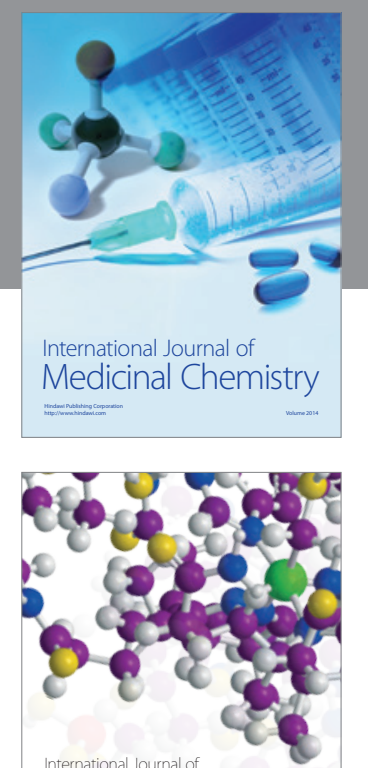

\section{Carbohydrate} Chemistry

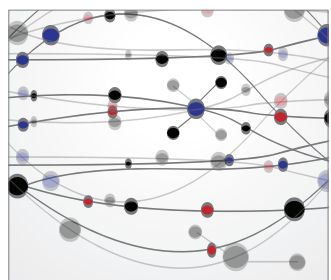

The Scientific World Journal
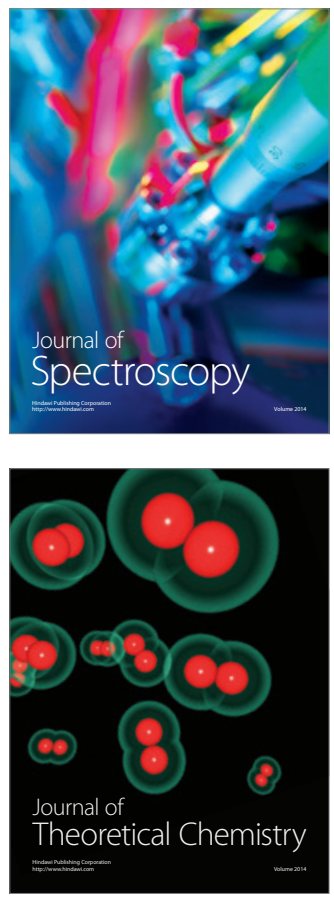
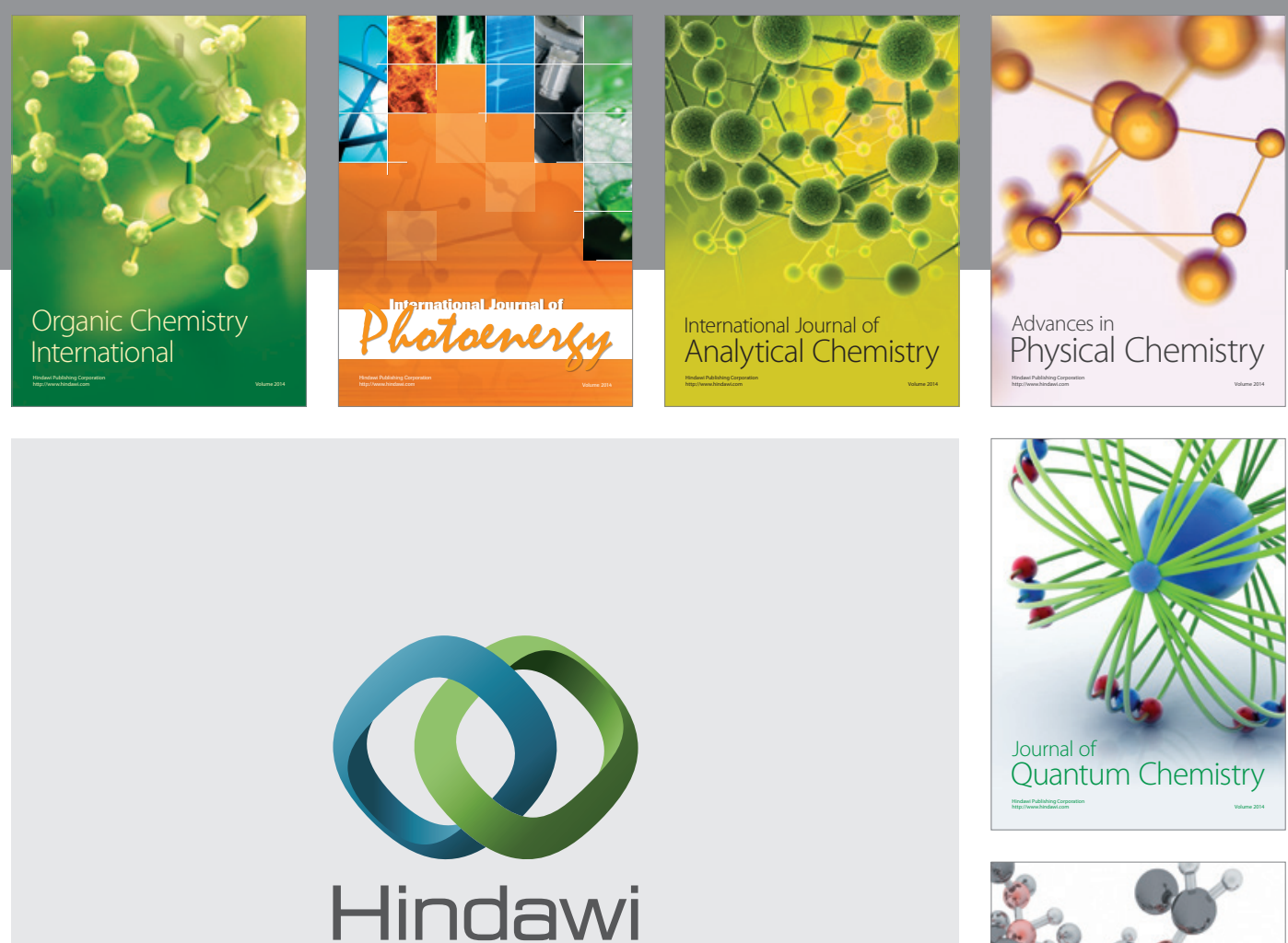

Submit your manuscripts at

http://www.hindawi.com

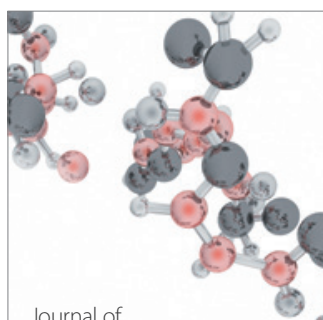

Analytical Methods

in Chemistry

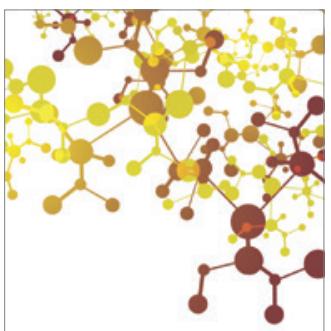

Journal of

Applied Chemistry

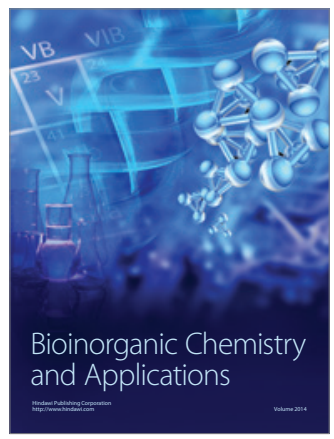

Inorganic Chemistry
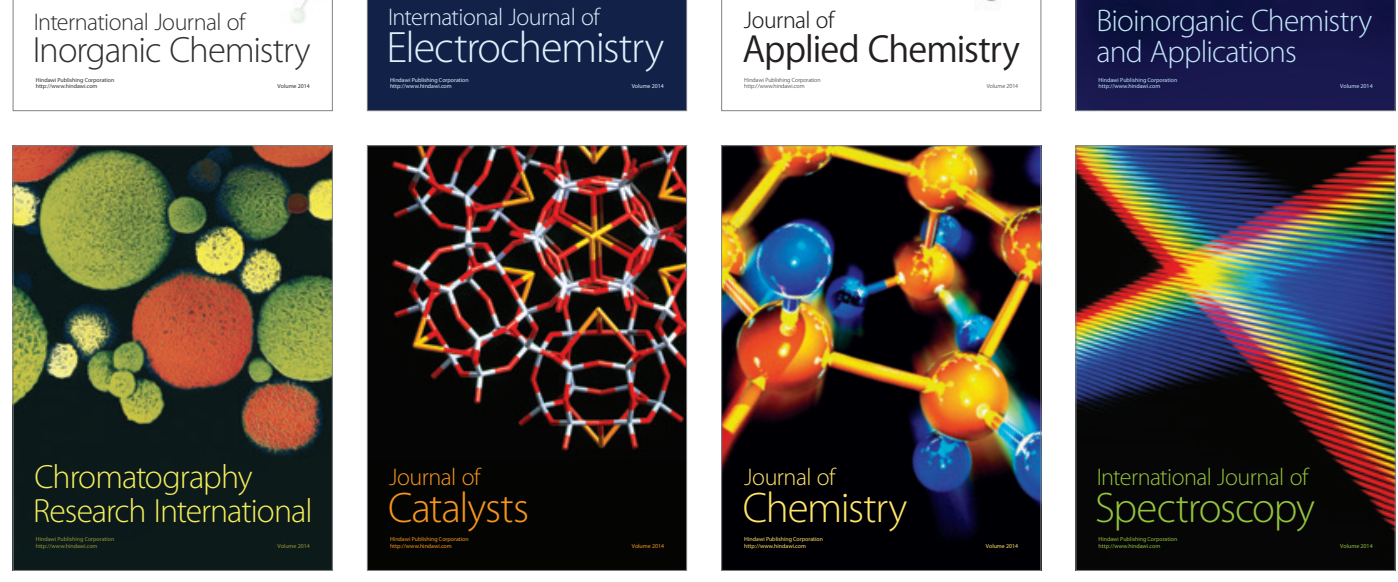